\title{
Integrated Avionics System Network Function Robustness Analysis based on Complex Network
}

\author{
Qiang $\mathrm{JI}^{1, \mathrm{a}}$, Qiming YANG ${ }^{2, \mathrm{~b}, *}$, Jiandong $\mathrm{ZHANG}^{2}$, Guoqing $\mathrm{SHI}^{2}$ \\ ${ }^{1}$ College of Aerospace Science and Engineering \\ National University of Defense Technology, Changsha 410073, China \\ ${ }^{2}$ The school of Electronics and Information \\ Northwestern Polytechnical University \\ Xi'an 710129, China \\ E-mail: ${ }^{\mathrm{j}}$ jiqheu@163.com \\ *E-mail: ${ }^{\mathrm{b}}$ yangqm1988@163.com \\ *Corresponding author
}

\begin{abstract}
Aiming at the phenomenon that only the local effect of the failure of the subsystem is considered on the study of robustness in the existing avionics network, this paper proposed a way to evaluate the robustness of the integrated avionics system using the efficiency of the subsystem. The method takes into account the global effect of the failure subsystem, and uses the efficiency of the subsystem to define the load, ultimate load and failure mode of each node. To give the optimization algorithm, it measures the robustness of the network by determining the ratio of the final failure subsystem in the network. Experimental analysis shows that the proposed method is feasible and effective for the integrated avionics system network.
\end{abstract}

Keywords: integrated avionics; complex networks; subsystem, robustness

\section{INTRODUCTION}

With the modularization of the structure of avionics system, the complex connection relationship between the subsystems can be naturally introduced as a network. At the macro level, the subsystem is regarded as a node, and the bus is regarded as a connecting edge. The theory of complex network and its dynamics provides a new analytical perspective and research method for the research of the structure and dynamics of the avionics system, alleviating and eliminating message blocking, optimizing the transmission efficiency, improving the security of information transmission [1].

Research shows that networks with different topological structures have different invulnerability against different ways of attack. Scale-free networks have a stronger fault tolerance than stochastic networks under random attack; but under intentional attacks, scale-free networks are rather fragile [2]. So it is quite a meaningful job to evaluate the robustness of complex networks. At present, the research method for complex networks robustness based on graph theory has attracted the attention of many scholars. Literature [3] and literature [4] determine the proportion of connected nodes as well as the shortest path between two nodes to measure the robustness of the network and conclude that the larger the proportion of connected nodes, also the shorter path between two nodes, the stronger the robustness of the network. In literature [5], the robustness of the network is measured using the degree of the decrease of the total network efficiency caused by the node deletion. Literatures [3-4] analyse the question from the structure of the network, without taking the effect of node failure on the function of the whole into consideration. The influence of the failure node on the adjacent nodes is analyzed in literature [5], but not in the global aspect of the network

This paper considers the global influence caused by the subsystem failure in the network. Firstly, we use the efficiency of the original avionics network subsystem to define the load and the ultimate load of each subsystem, according to the change of the load of the subsystem, the failure model of the subsystem is determined; Then the evaluation algorithm for the robustness of network function is given, and its optimization is carried out; Finally, the effectiveness and feasibility of the method are verified by experiments.

\section{ROBUSTNESS EVALUATION OF AVIONICS NETWORK}

\section{A. Theoretical Basis}

Suppose graph $G=(V, L)$ is a non - self - loop and undirected network, $V$ is a collection of all the nodes in the network; $L$ is a collection of edges in the network. $w_{i j}$ represents the weight of the connected nodes $i$ and $j$, the edge of the $l_{i j}$.

Definition 1: Node degree refers to the number of nodes that are directly connected to the node. At present, it is generally believed that the importance of the node has a bond relationship with its degree, therefore, the node degree can directly indicate the impact of the node on its adjacent nodes. The bigger the node degree is, the greater the importance of the node is to its adjacent nodes.

Definition 2: Node distance $d$ refers to the minimum value of the sum of all paths between the two nodes. If there is no path between node $i$ and node $j, d_{i j} \rightarrow \infty$.The maximum of the node distance in the network is the diameter of the network--- $R$.

Definition 3: Network efficiency $E$ refers to the average value of the reciprocal sum of the distances between all nodes in the network. 


$$
E=\frac{1}{n(n-1)} \sum_{i \neq j} \frac{1}{d_{i j}}
$$

There $n$ is the number of the network nodes. ${ }^{d_{i j}}$ is the distance between node $i$ and node $j$.

As can be seen from the definition of network efficiency $E$, network efficiency $E$ indicates the average closeness degree of all the node pairs in the network. The shorter the node distance is in the network, the higher the efficiency of the network is. In order to measure the average closeness degree between one node and other nodes in the network, we propose the concept of node efficiency $I$ based on the definition of the network efficiency.

Definition 4: Node efficiency $I_{k}$ refers to the average value of the reciprocal sum of distances between the node $k$ and the other nodes in the network. According to formula (1):

$$
E=\frac{1}{n(n-1)} \sum_{i \neq j} \frac{1}{d_{i j}}=\frac{1}{n} \sum_{k=1}^{n}\left(\frac{1}{n-1} \sum_{j=1, j \neq k}^{n} \frac{1}{d_{k j}}\right)
$$

Therefore, this paper defines $I_{k}$ as follows:

$$
I_{k}=\frac{1}{n-1} \sum_{i=1, i \neq k}^{n} \frac{1}{d_{k i}}
$$

It can be seen that the average efficiency of all nodes is the network efficiency $E$. The efficiency of a node indicates the average difficulty level of the node to the other nodes in the network. A higher node efficiency in the network indicates that it is easier to transmit information from this node to other nodes and fewer resource will be consumed in the process.

Definition 5: The majority of the nodes in the scale-free network are connected to a few links while some nodes (called HUB) have numerous links. Therefore, the topological potential of the nodes are of great difference. These highly connected nodes completely dominate the network connectivity and topology. Their distributions obey the power-law distribution and the network has a strong cohesion.

Definition 6: Stochastic network is a uniform network, its nodes couple randomly, which means no hub exist. The difference between the topology potential of each node is narrowed, the frequency distribution is approximated as Poisson distribution.

\section{B. Establishment of Model}

The robustness evaluation model of the function of avionics network studied in this paper is proposed after considering the connectivity and the maximum load of subsystem, where the load is raised aiming at the message flow of avionics network. Here, the load indicates the ability of subsystem to maintain its existing function. The heavier the subsystem load, the more intense the subsystem resource consumption is, the weaker the ability to maintain the existing functions.

Because the network efficiency $E$ is the average efficiency of all subsystems in avionics network, the efficiency of the subsystems also reflects the load of the subsystems to some extent. For the subsystems with fixed resource in the transmission process, if its efficiency is higher, it shows that the subsystem is closer to other subsystems in the network, the message transmission is easier, the resource consumed by transmission is less, the load of the subsystem is smaller. Therefore, in this paper, the subsystem efficiency is used to characterize the subsystem load $\mathrm{G}$, and the load of the subsystem $i$ is defined as follows:

$$
\mathrm{G}_{\mathrm{i}}=\exp \left(-I_{i}\right)
$$

For a subsystem, as long as it has a certain amount of resource to maintain its existing functions, it can withstand a certain amount of load. In this paper, the load that the subsystem can withstand in the network is defined as the tolerable load $C$ of the subsystem.For the avionics network, the resource available for each subsystem are fixed, the resource used by each subsystem in the message transmission process are the same. This causes that the loads they bear are the same---all are $C$. Therefore, this paper uses the same ${ }^{a}$ to represent the tolerable loads of network subsystems, and $C$ is defined as follows:

$$
\mathrm{G}_{\mathrm{i}}=\exp \left(-I_{i}\right)
$$

Where: $a \geq 1$ is the load tolerance coefficient of the nodes.

In the actual avionics network, the occurrence of the overload phenomenon in some subsystems doesn't lead to the collapse of the subsystem immediately, because the aircraft has automatic regulation ability to improve the subsystem capacity and relieve the subsystem load, which improves the whole reliability. Therefore, the failure of any subsystem in the network has a threshold which in this paper is defined as the limit load of subsystem:

$$
C_{\infty}=b \times C
$$

Where: $b \geq 1$ is the load limit coefficient of subsystem.

According to the subsystem tolerable load and limit load, we can define the failure probability $p_{i}$ of the subsystem $i$ as follows:

$$
p_{i}=\left\{\begin{array}{cc}
0 & G_{i}<C \\
\frac{G_{i}-C}{C_{\infty}-C} & C<G_{i}<C_{\infty} \\
1 & G_{i}>C_{\infty}
\end{array}\right.
$$


By (7) it can be seen that, when $a \rightarrow \infty, C \rightarrow \infty$, the system has enough resource to maintain the existing functions, and it isn't influenced by the changes of networks topology, which is equivalent to only studying the network robustness topology; when $a$ is fixed, $b \rightarrow \infty$, it shows that the limit load each subsystem can withstand is infinite, the failure of the subsystem is not entirely dependent on the subsystem load, and the subsystems maintain their existing function at a certain probability.

When some of the subsystems confront with a certain impact, the network message transmission path will be reconstructed, once the subsystem load exceeds its limit, the subsystem will breakdown, and it will in turn affect the load of other subsystems. After the network is attacked, the fewer the failures of the subsystem, the stronger the ability of the network to maintain its normal work is. Therefore, this paper uses the number of subsystem failures to measure the robustness of the network, and gives its normalized expression: $z z z$

$$
f=\frac{\sum_{i=1}^{N u m} f_{i}}{N u m \times n}
$$

Where: $n$ is the number of network subsystems, $N u m$ is the number of times that the network is attacked, and $f_{i}$ is the number of the failure subsystems caused by the attack. $i$

\section{Robustness Evaluation Algorithm for Avionics Network}

As can be seen from the above algorithm steps, the time complexity of the whole algorithm depends on the calculation of the shortest distance matrix $D=\left[d_{i j}\right]$ between different subsystems. As the time complexity of the Floyd algorithm is $O\left(n^{3}\right)$, in the worst case, the time complexity of the algorithm is $O\left(n^{4}\right)$. According to the characteristic of calculating the shortest distance between subsystems in Floyd algorithm, it can be optimized to reduce the time complexity. The Floyd algorithm needs to cycle the calculate edge weight matrix circularly $n$ times in the process of computing the shortest distance matrix $D=\left[d_{i j}\right]$,and when the shortest distances between all subsystems in the network have been found, we can notice that $D=\left[d_{i j}\right]$ will remain unchanged in the subsequent cycle processes. Therefore, in the process of solving $D=\left[d_{i j}\right]$, if the cycles are carried out blindly, it will cause the waste of computing resource and improve the time complexity of the algorithm.

This paper, in the process of calculating $D=\left[d_{i j}\right]$ by using the Floyd algorithm, determines whether the shortest distance between the subsystems has been found by setting the identification matrix. Once the shortest distance is found, the program will jump out of the cycle, thus reduce the running time and improve the efficiency of the algorithm. According to the random matrix theory, we know that the shortest distance between any pair of nodes can be obtained by the edge weight matrix $A=\left(a_{i j}\right)_{n \times n}$.

\section{EXPERIMENT AND ANALYSIS}

In the avionics network shown in Figure 1, the node degrees of node $v_{1}$ and node $v_{4}$ are 2 , if we only consider from the topology, when subsystem $v_{1}$ is invalid, it only shows the network subsystems are invalid whose node degrees are 2, the impact on the network should not be great. But we can see that the message transmission tasks connecting subnet $\mathrm{A}$ and $\mathrm{B}$ are all superimposed on subsystem $^{v_{4}}$, and if now the overload phenomenon occurs to $v_{4}$, the entire avionics network connectivity would be destroyed.

TABLE.1 ROBUSTNESS EVALUATION RESULTS OF AVIONICS NETWORK

\begin{tabular}{|c|c|c|c|}
\hline \multicolumn{2}{|c|}{$\begin{array}{l}\text { qualid nodes } \\
\text { quotas for evaluation }\end{array}$} & $v_{1}$ & $v_{1}, v_{2}$ \\
\hline \multirow[t]{2}{*}{$\begin{array}{l}\text { topological } \\
\text { robustness }\end{array}$} & $\begin{array}{c}\text { network } \\
\text { connectivit } \\
y\end{array}$ & $\begin{array}{c}\text { connecte } \\
\mathrm{d}\end{array}$ & $\begin{array}{c}\text { not } \\
\text { connecte } \\
\text { d }\end{array}$ \\
\hline & $\begin{array}{l}\text { maximal } \\
\text { cormected } \\
\text { subnet }\end{array}$ & 64 & 36 \\
\hline \multirow{2}{*}{$\begin{array}{c}\text { functional } \\
\text { robustness } \\
(a=1, \\
b=2 \text { ) }\end{array}$} & $\begin{array}{c}\text { network } \\
\text { connectivit } \\
y\end{array}$ & $\begin{array}{c}\text { not } \\
\text { connecte } \\
\text { d }\end{array}$ & $\begin{array}{c}\text { not } \\
\text { connecte } \\
\text { d }\end{array}$ \\
\hline & $\begin{array}{l}\text { maximal } \\
\text { cornected } \\
\text { subnet }\end{array}$ & 56 & 23 \\
\hline \multirow[t]{2}{*}{$\begin{array}{c}\text { functional } \\
\text { robustness } \\
(a=\infty)\end{array}$} & $\begin{array}{c}\text { network } \\
\text { connectivit } \\
\mathrm{y}\end{array}$ & $\begin{array}{c}\text { connecte } \\
\text { d }\end{array}$ & $\begin{array}{c}\text { not } \\
\text { connecte } \\
\text { d }\end{array}$ \\
\hline & $\begin{array}{l}\text { maximal } \\
\text { cormected } \\
\text { subnet }\end{array}$ & 60 & 40 \\
\hline
\end{tabular}

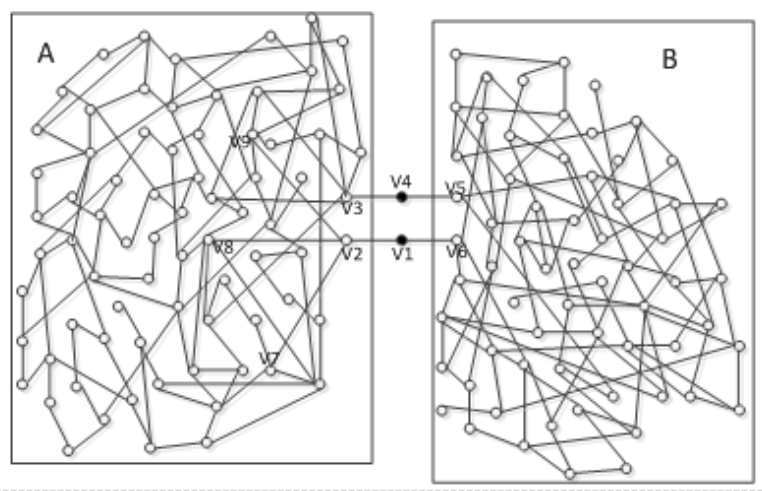

Figure.1 Sketch map of avionics network message flow

Therefore, in the actual avionics network robustness research process, just considering the topology structure is 
not enough, because the failures of systems will lead to the reallocation of the entire avionics network message flow, subsystem resource will be changed in the transmission process. For avionics network system, the failure of the subsystem will have a global impact on the function of the network. In the study of avionics network robustness, the load range and capacity constraint of subsystems also need to be considered.

This paper uses the network connectivity and the scale of the maximum connected subnet as the quotas to evaluate the network functional robustness and topological robustness under two kinds of situations---when $v_{1}$ fails or $v_{1}$ and $v_{2}$ fail. The results are shown in Table 1 .

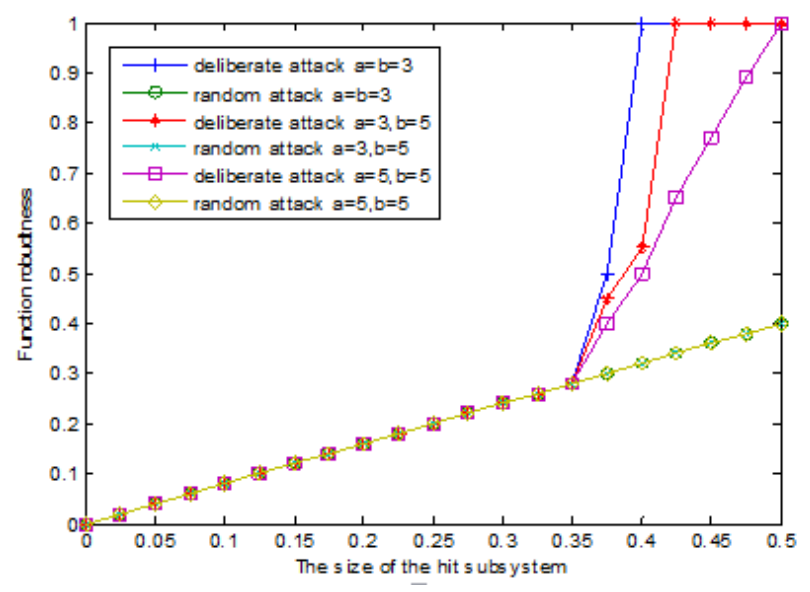

Figure.2 Sketch map of avionics network (with stochastic property) functional robustness

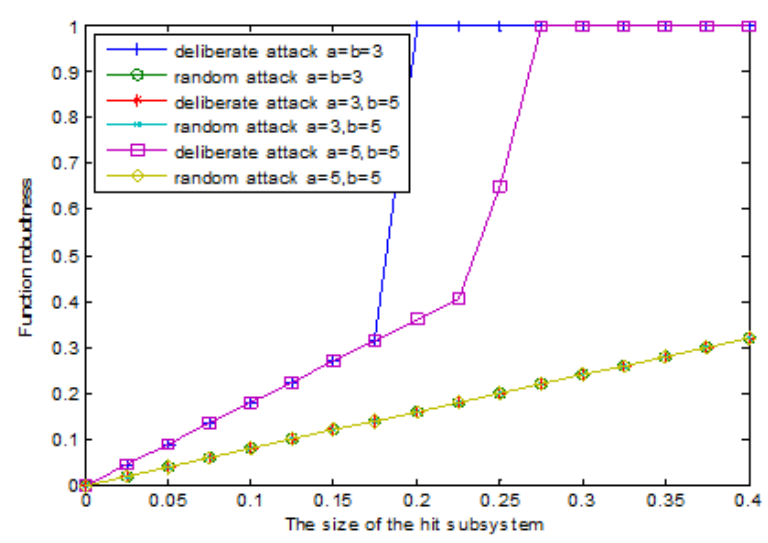

Figure.3 Sketch map of avionics network (with scale free property) functional robustness

It can be seen from Table 1 , when $a=\infty$, the evaluation results of network functional robustness are consistent with the evaluation results of its topological robustness; when $a=1, b=2$, the evaluation results of network functional robustness are not consistent with the evaluation results of its topological robustness.
With regard to the present research on complex networks robustness, it is generally considered that the scale free network has a stronger fault tolerance than the random network under random attacks; but under deliberate attacks, the scale free network is rather fragile. In order to compare with the existing conclusions, this paper considers the functional robustness of avionics network subsystem, constructs a scale free avionics network and a stochastic avionics network ---both with 300 subsystems and 700 edges. Then use the proposed method in this paper to evaluate their robustness. By simulation, we can get the simulation results of the scale free avionics network and the stochastic avionics network, as shown in Figure 2 and Figure 3.

It can be seen from Figure 2 and Figure 3, with the increases of the load tolerance coefficient $a$ and the load limit coefficient $b$ of the network nodes, the avionics network functional robustness becomes stronger gradually. When $a \rightarrow \infty$, the functional robustness curve of the avionics network is a straight line with a slope of 1 , which indicates that in the avionics network, overload will not lead to the failures of the subsystems, except for the subsystems being attacked, all other subsystems can maintain their existing message transmission abilities. Therefore, for the avionics network system, we need to improve the system reliability and reduce the number of the failure subsystems caused by overload. We can increase the message processing resource of the subsystems to improve their capacities and reduce the probability of function failure,. Thereby the network has a stronger robustness and the system reliability is enhanced.

\section{CONCLUSIONS}

In view of the existing avionics network robustness research, this paper only considers local effects of the subsystem failure and the defects of robustness, proposes a method for evaluating network robustness by considering the global information and using the subsystem efficiency, and gives the optimization algorithm. The experiment shows that only using the topological robustness to evaluate the avionics network robustness is not enough, the subsystem tolerable load should also be considered, we can increase the message processing resource of subsystems to improve the reliability of the actual system; For the measurement of the real network functional robustness, it should be restricted by the condition under which the system could maintain its normal functions.

\section{ACKNOWLEDGMENTS}

The work was supported by the Science and Technology on Avionics Integration Laboratory. The gratitude goes to all the staff of the Foundation.

\section{REFERENCES}

[1] Du Wenbo. Research on Complex Network and Network Dynamics for Air Transportation Systems [C].Hefei: University of Science and Technology of China, A dissertation for doctor's degree, 2010.

[2] Bao Z J, Cao Y J, Ding L J, et al. Comparison of cascading failures in small-world and scale networks subject to vertex and edge attacks [J]. Physica A: Statistical Mechanics and its Applications, 2009, 338(20): 4491-4498. 
[3] Wu J, Barahona M, Tan Y J, et al. Spectral measure of structural robustness in compex network[J]. IEEE Transactions on Systems Man and Cybernetics Part A, 2011, 41(6):1244 1252.

[4] Wu J, Barahona M, Tan Y J, et al. Natural connectivity of complex network[J]. Chin Phys Lett, 2010,27(7):078902.

[5] Wang Lin, Dai Guan. Scale-free property, Scale-free phenomenon and their control in complex networks [M].Beijing: Science Press,2009:137. 